or capecitabine (EOX, $n=244)$. The unadjusted hazard ratio (HR) for death when capecitabinecontaining regimens were compared with fluorouracil-containing regimens was 0.86 (95\% $\mathrm{Cl}$ 0.80-0.99), while the unadjusted HR for death in a comparison of oxaliplatin-containing and cisplatin-containing regimens was 0.92 (95\% Cl 0.80-1.10). Two-by-two comparison showed noninferiority for both capecitabine and oxaliplatin. The 1-year survival rates in the ECF, ECX, EOF, and EOX groups were $37.7 \%, 40.8 \%$, $40.4 \%$, and $46.8 \%$, respectively, while the median survival times were 9.9 months, 9.9 months, 9.3 months and 11.2 months. Overall survival was longer with EOX than with ECF (HR for death $0.80,95 \% \mathrm{Cl} 0.66-0.97)$. Progression-free survival and the overall response rates did not differ significantly between the groups. Compared with cisplatin, oxaliplatin was associated with fewer incidences of grade 3-4 neutropenia, alopecia, renal toxic events and thromboembolism but with higher incidences of grade 3-4 diarrhea and neuropathy. Capecitabine and fluorouracil had similar toxicity profiles.

This study showed that with respect to overall survival, capecitabine and oxaliplatin are at least as effective as fluorouracil and cisplatin, respectively, for the treatment of esophagogastric cancer.

Original article Cunningham D et al. (2008) Capecitabine and oxaliplatin for advanced esophagogastric cancer. $N$ Engl J Med 358: 36-46

\section{Chemoradiotherapy: standard treatment for Hodgkin's disease with favorable prognosis}

The European Organisation for Research and Treatment of Cancer Lymphoma Group H7 trial results led to the proposal that chemotherapy followed by radiotherapy should be the standard treatment for Hodgkin's lymphoma. On the basis of the prognostic factors used in the $\mathrm{H} 7$ trial, Fermé et al. have compared different treatment regimens for patients with untreated stage I or II supradiaphragmatic Hodgkin's lymphoma.

The trial included 1,538 patients with favorable (H8-F trial) or unfavorable ( $\mathrm{H} 8-\mathrm{U}$ trial) prognostic features. In the H8-F trial, 272 patients were randomized to receive subtotal nodal radiotherapy (control group), while 270 received three cycles of the MOPP-ABV hybrid regimen (mechlorethamine, vincristine, procarbazine and prednisone together with doxorubicin, bleomycin and vinblastine) plus involved-field radiotherapy. The 10-year overall survival rate for patients who received radiotherapy was $92 \%$, compared with $97 \%$ for patients who received chemotherapy plus radiotherapy $(P=0.001)$. The corresponding 10-year event-free survival rates were $68 \%$ and $93 \%(P<0.001)$. In the $\mathrm{H} 8-\mathrm{U}$ trial, 336 patients received six cycles of MOPP-ABV plus involved-field radiotherapy (group A), 333 patients received four cycles of MOPP-ABV plus involved-field radiotherapy (group B) and 327 patients received four cycles of MOPP-ABV plus subtotal nodal radiotherapy (group C). The 10-year overall survival rates for groups A, B, and $\mathrm{C}$ were $88 \%, 85 \%$ and $84 \%$, respectively, while the corresponding 10-year event-free survival estimates were $82 \%, 80 \%$ and $80 \%$.

The authors comment that for patients with supradiaphragmatic early-stage Hodgkin's lymphoma, chemotherapy plus involved-field radiotherapy should be the standard therapy-three cycles should be given to patients with favorable prognosis, whereas patients with unfavorable prognosis should receive four cycles.

Original article Fermé C et al. (2008) Chemotherapy plus involved-field radiation in early-stage Hodgkin's disease. N Engl J Med 357: 1916-1927

\section{Prognostic value of pretreatment quality of life in patients with head and neck cancer}

Pretreatment sociodemographic variables can predict outcome in patients with cancer and could, therefore, be used to tailor therapy. Siddiqui et al. analyzed data from two phase III studies of head and neck cancer to assess the predictive value of baseline health-related quality of life (HRQOL) on outcome.

The two randomized Radiation Therapy Oncology Group trials on head and neck cancer enrolled patients with locally advanced stage II-IV squamous cell carcinoma of the head and neck and a Karnofsky performance status of $\geq 60$. Patients completed the Functional Assessment of Cancer Therapy-Head and Neck, version 2 (FACT-H\&N) HRQOL questionnaire before undergoing radiation therapy with or without concurrent chemotherapy.

Pretreatment FACT-H\&N data were available for 1,093 patients, of whom 501 (46\%) experienced locoregional failure and 646 (59\%) died during 